\title{
An Arabidopsis thaliana (Ler) inbred line exhibiting stacked stem/inflorescences mainly due to the reduced AP1 expression
}

\author{
Xiaoli Qi ${ }^{1}$, Yao Jiang ${ }^{2}$, Fang Tang ${ }^{2}$, Shu-Tang Zhao ${ }^{2}$, Meng-Zhu Lu ${ }^{*}$ \\ From IUFRO Tree Biotechnology Conference 2011: From Genomes to Integration and Delivery \\ Arraial d'Ajuda, Bahia, Brazil. 26 June - 2 July 2011
}

\section{Background}

Bolting is regarded as the initiation of reproduction stage in the growth and development of an Arabidopsis plant, when a set of floral integrator genes activate the expression of floral meristem identity genes $L F Y$ and $A P 1$ to initiate flowering transition[1,2]. However, how the expression of key genes, such as $A P 1$, responds of diverse signals during flower development remains largely unknown. Here we have obtained an inbred line exhibiting an abnormal stem/inflorescence and flower development.

\section{Methods}

The morphological variations were carefully observed visually or with a Zeiss SteREO Discovery V8 stereomicroscope. Hybridization of GeneChip arrays was done in an Affymetrix Hybridization Oven 640 following the manufacturer's protocol (Affymetrix) and expression levels in seedlings and flower buds were calculated from Affymetrix intensity data. Real-time PCR was performed on an ABI 7500 Real-Time PCR System (Applied Biosystems, Shanghai, China) with SYBR GreenPCR Master Mix (Applied Biosystems) as the fluorescence source.

\section{Results}

The inbred line exhibits a flower phenotype similar to the ap1, such as homeotic conversion of sepals (first whorl) to leaf-like, petals often absent (second whorl) and complete flower-to-inflorescence conversions (Figure1, B). However flower meristem was replaced by emerging inflorescence meristems thus leading to a

\footnotetext{
* Correspondence: lumz@caf.ac.cn

${ }^{2}$ State Key Lab of Forest Genetics and Tree Breeding, Chinese Academy of Forestry, Beijing 100091, China

Full list of author information is available at the end of the article
}

stacked stem/inflorescences before final flowering (Figure1, D). Position and degree of stacked stem/inflorescences are varied differently (Figure1, E and F).

Microarray and quantitative real-time PCR analysis revealed that the expression of AP1 was significantly reduced, while the expression of its interacting genes TERMINAL FLOWER 1 (TFL1), OVEREXPRESSION OF CONSTANS(SOC1), AGAMOUS-like 24 (AGL24), SEPALLATA (SEP) and upstream genes FLOWERING LOCUS $M$ (FLM) were increased in flower buds (Figure2).

$A P 1$ sequence analysis showed that the promoter, coding sequences and intron splice sites of $A P 1$ genomic sequence in this inbred line were unchanged comparing to that in wildtype, suggesting the complexity in the regulation of AP1 in the line. Therefore these synthetic contributions caused the development of this unique phenotype. For instance, TFL1 was found to be highly expressed, and this gene can negatively regulate AP1 and specify inflorescence meristem identity leading to a delay of floral meristem formation[3]. On the other hand, the low levels of AP1 in flower buds cannot repress expression of $A G L 24$ and SOC1, which promote inflorescence fates rather than flower formation in the meristem and result in more abundant and longer inflorescences[4]. This expression variation of these genes is subjected to a threshold[4], leading to an $\mathrm{ON} /$ OFF expression pattern of the master regulatory gene(s) (like AP1) to specify a floral or a stem/inflorescence meristem.

\section{Conclusions}

The inbred line identified in this study is phenotypically similar to ap1 mutants with noticeable deviations. The abnormal stem/inflorescence of the inbred line was 


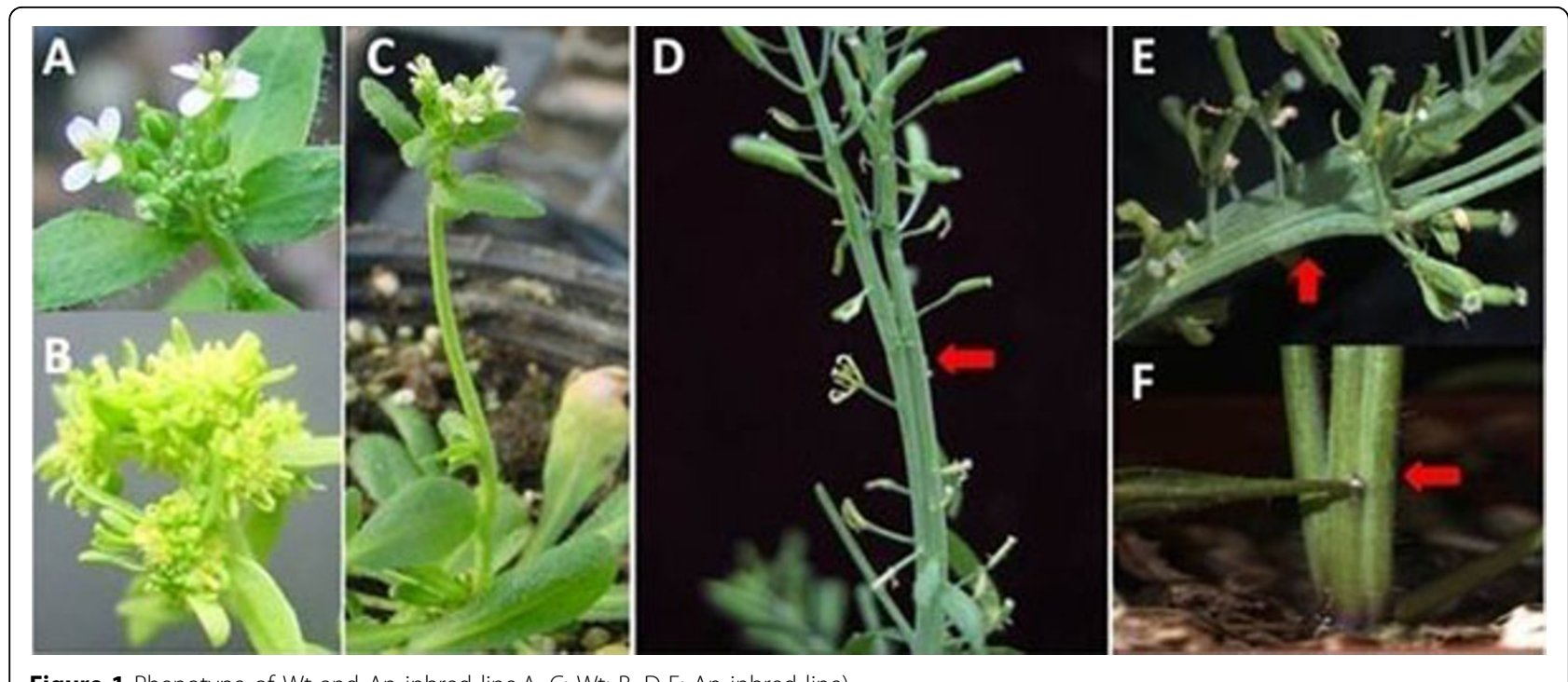

Figure 1 Phenotype of Wt and An inbred line.A, C: Wt; B, D-F: An inbred line)

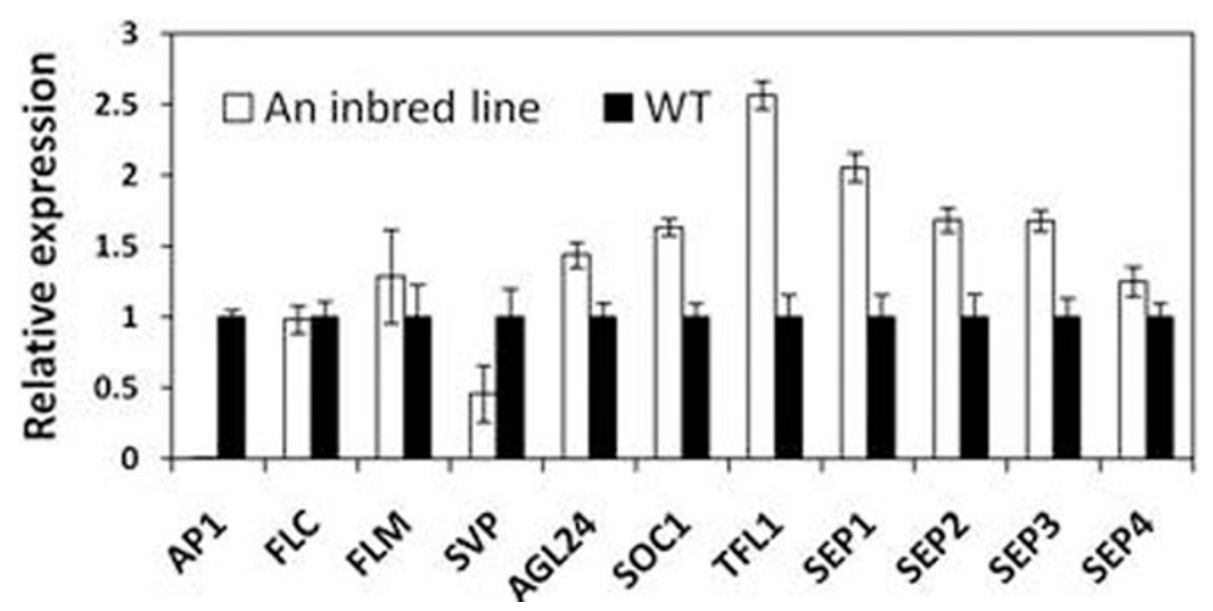

Figure 2 Expression of flowering regulatory genes in flower buds using qRT-PCR

mainly caused by significant downregulation of $A P 1$, but also is attributable to crosstalk among key genes like TFL1, AGL24, SOC1, etc. to control the transition of vegetative growth to the flowering phase. The inbred linemerits further molecular characterization to understand better the regulatory molecular network.

\section{Acknowledgements}

Supported by the national Natural Scienve Foundation of China (30771697), the Research Foundation of Jiamusi University (S2010-53) and the Education Department of Heilongjiang Province (12513091)

\section{Author details}

'College of Life Sciences, Jiamusi University, Jiamusi 154007, China. ${ }^{2}$ State Key Lab of Forest Genetics and Tree Breeding, Chinese Academy of Forestry, Beijing 100091, China.
Published: 13 September 2011

\section{References}

1. Blazquez M, Weigel D: Integration of oral inductive signals in Arabidopsis. Nature 2000, 404:889-892.

2. Borner R, Kampmann G, Chandler J, Glei ner R, Wisman E, Apel K, Melzer S: A MADS domain gene involved in the transition toflowering in Arabidopsis. Plant J 2000, 24:591-599.

3. Liljegren S, Gustafson-Brown C, Pinyopich A, Ditta G, Yanofsky M: Interactions among APETALA1,LEAFY, and TERMINAL FLOWER1 specify meristem fate. The Plant Cell 1999, 11:1007-1018.

4. Liu C, Xi W, Shen L, Tan C, Yu H: Regulation of floral patterning by flowering time genes. Dev Cell 2009, 16:711-722.

5. Raj A, Rifkin $S$, Andersen E, Van Oudenaarden A: Variability in gene expression underlies incomplete penetrance. Nature 2010, 463:913-918.

doi:10.1186/1753-6561-5-S7-P68

Cite this article as: Oi et al: An Arabidopsis thaliana (Ler) inbred line exhibiting stacked stem/inflorescences mainly due to the reduced AP1 expression. BMC Proceedings 2011 5(Suppl 7):P68. 